\title{
Philosophiques
}

\section{Réponses à mes critiques}

\section{Richard G. Delisle}

Volume 38, numéro 1, printemps 2011

Les conférences Hugues Leblanc 2010

URI : https://id.erudit.org/iderudit/1005730ar

DOI : https://doi.org/10.7202/1005730ar

Aller au sommaire du numéro

Éditeur(s)

Société de philosophie du Québec

ISSN

0316-2923 (imprimé)

1492-1391 (numérique)

Découvrir la revue

Citer ce document

Delisle, R. G. (2011). Réponses à mes critiques. Philosophiques, 38(1), 285-303.

https://doi.org/10.7202/1005730ar d'utilisation que vous pouvez consulter en ligne.

https://apropos.erudit.org/fr/usagers/politique-dutilisation/ 


\title{
Réponses à mes critiques
}

\author{
RICHARD G. DELISLE \\ Université de Lethbridge \\ richard.delisle@uleth.ca
}

\begin{abstract}
À la lumière des critiques émises par Daniel Becquemont, Barbara Continenza et Michel Morange, la discussion qui suit s'organise autour des axes principaux suivants : 1) la fondation du darwinisme dans ses rapports avec la physique newtonienne; 2) la nature de l'entité de recherche darwinienne; et 3 ) les rapports du darwinisme avec la révolution transformiste universelle. Du fait que l'une des thèses principales de l'ouvrage interpelle explicitememt la notion kuhnienne de stage disciplinaire "pré-paradigmatique ", l'ensemble de cette réflexion comporte pour toile de fond l'épistémologie historique.
\end{abstract}

\section{Réponse au commentaire de Daniel Becquemont}

La question de l'ancrage épistémologique des travaux de Charles Darwin a mobilisé par le passé beaucoup d'attention. Daniel Becquemont revient sur l'ensemble de cette problématique par le biais des rapports que le fondateur du darwinisme entretient à l'endroit de la révolution scientifique et des disciplines physiques. Celui-ci est concerné par la difficulté à établir la véritable place de Darwin dans l'historiographie à la suite d'interprétations divergentes. Pour ma part, j'avance que ces divergences de points de vue sont plus superficielles qu'il n'y paraît, permettant ainsi de contourner cet obstacle.

\section{Charles Darwin et la révolution scientifique}

De nombreux travaux portant sur les écrits de Charles Darwin ont mis en lumière ses choix épistémologiques, notamment les prescriptions méthodologiques auxquelles il tentait de s'astreindre afin d'élaborer la meilleure des sciences qui puisse se faire selon les canons de la science victorienne. Comme le relève justement Daniel Becquemont, les commentateurs modernes ne sont pas tous en parfait accord quant aux véritables réalisations épistémologiques de Darwin:

Si de nombreux spécialistes de Darwin considèrent que celui-ci s'insère effectivement dans les canons de ce qu'on appelait alors la méthode inductive, ce que Darwin lui-même revendiquait, d'autres ont plutôt mis l'accent sur une certaine rupture de la théorie darwinienne avec l'épistémologie de la révolution scientifique, en ce qui concerne la prédiction, la nature du «fait scientifique » et le rôle de l'analogie.

À mon sens, cette apparente opposition dans l'interprétation des réalisations darwiniennes n'entame aucunement ma thèse selon laquelle Darwin 
et ses quelques véritables successeurs dont Simpson et Mayr se positionnent en prolongement de la révolution scientifique. Cette opposition, me semblet-il, dissimule une unité plus fondamentale. Certes, certains choix issus de la phase initiale de la révolution scientifique lors des $\mathrm{XVII}^{\mathrm{e}}$ et $\mathrm{XVIII}^{\mathrm{e}}$ siècles, dont l'universalisme des lois, le réductionnisme physicaliste et le déterminisme étaient tout à fait inadaptés à la réalité " historique » et "contingente » de la biologie de l'évolution, une spécificité que Darwin a non seulement su reconnaître mais mettre en œuvre mieux que quiconque avant lui. Mais cela ne fait pas de Darwin et de ses émules des penseurs s'inscrivant à l'extérieur de cette tradition épistémologique puisque la révolution scientifique portait en son sein des choix ou des potentialités conceptuelles suffisamment «neutres » pouvant s'appliquer avec profit à la biologie de l'évolution. J'insisterai sur trois de ces choix uniquement:

1) L'actualisme ou une science établie sur la connaissance des mécanismes et des processus présentement en cours. Quoique la réalité évolutive comporte en elle-même une forte dimension historique - d'où la contribution significative de la paléontologie avant la publication de L'origine des espèces en 1859 et les soucis qu'elle causera à Darwin - c'est le propre de Darwin d'avoir ancré sa théorie dans une effectivité toute actuelle. Ce n'est pas par hasard si L'origine des espèces s'ouvre sur un chapitre portant sur la sélection artificielle, un processus pouvant être directement observé. Si les éleveurs parviennent à modifier les espèces en peu de temps, insiste-t-il, il nous suffit d'imaginer les effets d'une sélection naturelle qui, d'une part, possède à sa disposition des durées prolongées et, d'autre part, bénéficie d'une puissance d'action incomparable du fait qu'elle scrute les moindres variations des organismes à tout moment ${ }^{1}$. Le noyau explicatif de la théorie darwinienne est entièrement fondé dans l'actualisme, les manifestations évolutives passées s'expliquant par la simple extrapolation des effets observés actuellement et dont l'ampleur se trouve amplifiée uniquement par la durée géologique et non par la modulation du mécanisme au cours du temps. C'est ainsi que Darwin répondait à la prescription du newtonianisme qui réclamait une science des «causes vraies " dont les deux composantes principales sont: a) la formulation de causes qui se doivent d'exister réellement et non pas être supposées exister en l'absence de preuves tangibles; b) la formulation de causes suffisantes pouvant produire les effets postulés. Alors que l'analogie entre la sélection artificielle et la sélection naturelle répondait à la première exigence, l'argument extrapolationniste sur la durée géologique s'adressait à la deuxième.

1. Darwin, 1859 , p. $61,80,82-84$. 
2) Une structure explicative compacte s'approchant de l'idéal axiomatico-déductif dont le pouvoir explicatif puisse s'étendre à de nombreux phénomènes. Le newtonianisme, rappelons-le, a été compris par les philosophes des $\mathrm{XVIII}^{\mathrm{e}}$ et $\mathrm{XIX}^{\mathrm{e}}$ siècles dont Thomas Reid, William Whewell et John Herschel comme étant élaboré, par exemple, selon la méthode de l'observation des orbites célestes (induction) menant à la postulation de la loi de la gravitation (généralisation), elle-même servant de base explicative à la prédiction d'autres phénomènes qui sont, en apparence seulement, d'une nature autre, dont la chute des corps et le cycle des marées (déduction). Souscrivant à cette méthode scientifique, Darwin expose dans les chapitres 1 et 2 de L'origine des espèces la partie inductive et sa généralisation dans l'analogie entre la sélection artificielle (observation) et la sélection naturelle (loi), suivie dans les chapitres 9 à 13 de l'explication de phénomènes aussi divers que ceux observés en paléontologie, en biogéographie, en morphologie, en embryologie, etc. Je ne défends pas ici l'idée que Darwin soit parvenu à sa théorie par la voie de la méthode inductive ${ }^{2}$, mais simplement qu'il a eu le souci dans son œuvre maîtresse de présenter la structure inductivo-déductive de son argumentation. Certes, il est impossible pour Darwin de proposer une déduction de nature prédictive du fait même de la nature historique et contingente de l'évolution biologique, comme le remarque David Hull ${ }^{3}$. Mais c'est précisément là où Darwin innove en élargissant et en assouplissant les prescriptions épistémologiques du newtonianisme, indépendamment des incompréhensions de Whewell et Herschel à l'endroit de la nouvelle réalité de l'évolution.

3) Une ontologie corpusculaire s'opposant au holisme des entités supérieures. La révolution scientifique portait en elle un autre enseignement riche en valeur heuristique et conceptuelle pour Darwin. La physique et la chimie mécanistes allaient s'élaborer sur le principe de l'atomisme, soit la supposition que l'ensemble des phénomènes physico-chimiques trouve dans la relation de leurs entités constitutives les plus infimes et invisibles la cause ultime des manifestations phénoménologiques. Même Newton, qui pourtant s'affichait contre la postulation d'entités "hypothétiques" (spéculatives) - au sens critique de cette expression - reconnaissait que la science du microscopique en viendrait certainement à révéler la réalité atomique. Les atomes sont pour la physique et la chimie mécanistes ce que les organismes biologiques seront pour la biologie de Darwin. Dans les deux cas il s'agit d'un réductionnisme corpusculaire mais s'appliquant à des niveaux différents de la hiérarchie de la matière. Alors que les phénomènes

2. Becquemont, 2009, p. 12.

3. Hull, 1973, p. 32. 
physico-chimiques de nature macroscopique se ramènent à des causalités microscopiques, de même les manifestations macroévolutives de la hiérarchie linnéenne se situant aux niveaux des genres, des familles, des ordres, des classes, des embranchements et des domaines, se ramènent à une efficace évolutive jouant uniquement sur les entités inférieures dont l'organisme individuel et l'espèce. Il est en effet remarquable, à la lecture de L'origine des espèces, de constater que Darwin applique l'action de la sélection naturelle uniquement à des entités se situant entre l'organisme individuel, la sous-espèce et l'espèce. Les réalités dites supérieures ne sont, pour Darwin, que des épiphénomènes d'une causalité agissant aux niveaux les plus inférieurs de la hiérarchie, et dont l'ampleur ne s'explique que par l'argument extrapolationniste dans la durée géologique.

On le voit, la théorie darwinienne puisera dans la révolution scientifique des enseignements épistémologiques dont l'utilisation sera véritablement constitutive. Seulement, il sera nécessaire pour Darwin d'en élargir les applications en souscrivant davantage à l'esprit qu'à la lettre de cette révolution.

\section{Le darwinisme et le paradigme de la physique}

La proximité décrite plus haut entre la physique et l'évolutionnisme de Charles Darwin pousse Daniel Becquemont à s'interroger sur la nature réelle de l'utilisation faite de ce rapprochement dans mon ouvrage:

Richard Delisle ne prête-t-il pas, dans un souci de mieux contraster la pensée «immature» du néodarwinisme, une trop grande cohérence à la physique, pratiquement assimilée à "l'esprit de la révolution scientifique" ? Ceci, depuis les débuts de la révolution scientifique jusqu'à nos jours? S'agit-il d'un mode de présentation rhétorique pour mieux souligner la thèse centrale, que la théorie de l'évolution en est encore à un stade "préparadigmatique »?

D'abord, comme nous l'avons vu, la théorie darwinienne est véritablement constitutive des prescriptions épistémologiques émanant de la Synthèse newtonienne. Loin d'être le fait de Darwin seul, l'idée de l'érection d'une véritable synthèse en biologie de l'évolution à l'image de la Synthèse newtonienne est reprise dans les années 1930 et $1940^{4}$, d'où l'appellation de "synthèse moderne de l'évolution » pour la qualifier. Sur le plan structurel comme sur le plan étiologique, les deux synthèses se présentent de la même manière: la théorie de la gravitation et la théorie de la sélection naturelle constituent un noyau explicatif rendant compte d'une foule de manifestations phénoménologiques, qu'il s'agisse de la révolution des planètes, des cycles des marées ou de la chute des corps, ou encore de la cladogenèse, de l'anagenèse ou de comportements adaptatifs. La notion d'un rapproche-

4. Smocovitis, 1996, p. 171. 
ment entre la Synthèse newtonienne et la Synthèse moderne de l'évolution possède un véritablement fondement historique.

Le questionnement de Daniel Becquemont ouvre sur d'autres considérations plus subtiles encore. Que la physique ne constitue pas un champ d'investigation présentant une grande cohérence est un fait que j'accepte sans aucune autre forme de procès. Toutefois, la question des rapports des néo-darwiniens avec la physique se pose en d'autres termes: ceux-ci conçoivent le darwinisme comme un champ d'investigation centré exclusivement autour de la théorie sélective. Alors que l'ensemble du champ de la physique incarne une constellation de disciplines et de théories (physique quantique, physique relativiste, théorie du chaos, etc.), le darwinisme tel que conçu dans l'esprit même des néo-darwiniens ne présente aucune commune mesure avec le pluralisme inhérent de la physique. Voilà pourquoi ceux-ci procéderont plus volontiers à une association avec une physique newtonienne bien circonscrite, plus qu'avec une programmation de recherche diffuse que représente l'ensemble du champ physique.

Cette première précision nous amène sur le terrain de la rhétorique, celle dont font preuve certains néo-darwiniens à l'endroit de la théorie darwinienne. Si ceux-ci se montrent explicitement fidèles à l'endroit d'une Synthèse moderne d'inspiration toute darwinienne, il n'empêche qu'une analyse plus approfondie de leurs contributions évolutionnistes révèle l'étroitesse de ce cadre théorique pour accommoder leurs visions fort divergentes (cette idée sera développée plus avant ci-dessous) ${ }^{5}$. Il semble donc que l'allégeance darwinienne de nos auteurs soit plus de façade qu'il n'y paraît. C'est dans la foulée de ce constat que je reprends le concept kuhnien d'un darwinisme toujours à un stade pré-paradigmatique : période durant laquelle plusieurs métaphysiques rivalisent pour fonder le même champ disciplinaire, voire la même théorie ${ }^{6}$. Ici, je m'intéresse moins à la justesse de l'ensemble de l'épistémologie de Thomas Kuhn qu'à l'utilité de certains de ses concepts pour éclairer une opposition de vues parmi les évolutionnistes, et dont l'ampleur était tout simplement insoupçonnée. Dans ce contexte, il me semble légitime de soulever la question de la compatibilité ou de l'incommensurabilité de toutes ces visions évolutionnistes au cœur d'une théorie darwinienne traditionnellement comprise comme centrée autour d'une théorie sélective conceptuellement compacte. Daniel Becquemont a raison de parler de rhétorique, toutefois celle-ci est à débusquer non dans ma thèse mais dans les rapports que certains néo-darwiniens entretiennent à l'endroit de la théorie darwinienne elle-même.

Est-ce le rôle du philosophe de mettre en lumière ces tensions argumentatives internes? Daniel Becquemont soulève les questions suivantes:

5. Voir aussi Delisle, 2011.

6. Kuhn, 1970, p. 11, 17-20, 23-24. 
Mais le souci des philosophes de déceler des contradictions et d'aspirer à une cohérence parfaite n'est-il pas dénaturant? [...] La philosophie de Delisle, ici, n'est-elle pas trop exigeante et ne demande-t-elle pas trop à la pensée humaine? Dire que c'est une pensée pré-paradigmatique n'est-il pas alors un acte de foi en un moment idéal et imaginaire où la biologie néodarwinienne se constituerait par la suite comme sans contradiction comme un nouveau paradigme, analogue dans sa solidité à l'idéal newtonien... qui pourtant reposait sur la métaphysique de la religion naturelle?

Quoique la philosophie soit, à raison ou à tort, parfois animée par un idéal normatif, l'esprit de l'ouvrage dont il est question ici ne répond aucunement à cet impératif. La question de l'incommensurabilité de plusieurs métaphysiques lors d'une phase pré-paradigmatique me semble importante, car potentiellement révélatrice d'une théorie darwinienne qui n'a pas une unique assise, et ce, malgré les choix de Darwin (et certains successeurs) de s'inspirer fortement de la Synthèse newtonienne. Le cas échéant, cela constituerait un puissant indicateur selon lequel la théorie sélective darwinienne est, conceptuellement parlant, suffisamment «neutre» pour être insérée dans des cadres interprétatifs différents. Ainsi, non seulement il serait historiquement faux de concevoir les études darwiniennes comme s'insérant au sein d'une seule tradition légitime de recherche, mais de plus le darwinisme serait moins prisonnier qu'il n'y paraît des choix de son fondateur puisqu'ouvert sur un avenir encore indéterminé. Les tentatives de fonder la théorie sélective darwinienne sur des cadres épistémologico-métaphysiques concurrents me semblent caractérisées par une difficile cohabitation, précisément parce que ceux-ci débouchent sur des implications évolutionnistes contradictoires, d'où la notion de leur incommensurabilité. Afin d'éviter certaines répétitions, des développements ultérieurs viendront étayer l'argumentation entamée ici.

\section{Réponse au commentaire de Barbara Continenza}

Les considérations autour du darwinisme débouchent souvent sur la question de sa nature intrinsèque. Grâce au commentaire de Barbara Continenza, la réflexion est d'emblée placée sur le terrain d'un darwinisme compris comme un programme de recherche, soit une entité conçue en perpétuelle transformation. Je suis très à l'aise avec cette caractérisation, quoique je propose pour ma part une variante de ce modèle de développement scientifique.

\section{Le darwinisme: un programme de recherche au développement de plus en plus inclusif?}

S'appuyant sur les travaux du biologiste du développement, Conrad Waddington, à titre d'exemple, Barbara Continenza développe l'intéressante thèse avancée par Mayr selon laquelle le programme de recherche darwinien aurait la propriété de se faire de plus en plus inclusif alors qu'il se déploie 
dans la durée: «Mais si le programme de la «synthèse» fut un programme de recherche "étroit", ne pourrait-on pas soutenir qu'il le fut même au nom d'une praticabilité des programmes de recherche [...].» Selon cette thèse, certains enjeux scientifiques se présentent de manière trop précoce dans l'histoire d'un programme de recherche pour véritablement trouver une résolution à ce moment-là. Il est donc préférable de les laisser de côté afin de se concentrer sur les problèmes présentement solubles, avec l'espoir que ces enjeux difficiles trouveront éventuellement réponse dans une phase ultérieure du programme de recherche. Cette thèse se fait l'écho d'une des idées phares des «programmes méthodologiques de recherche» de Imre Lakatos: la protection du noyau théorique au cœur d'un programme de recherche dans le but d'éviter sa réfutation inutilement précoce. C'est ainsi que certains aspects de la biologie développementale proposés par Waddington, et jadis situés à l'extérieur du "paradigme », puisque problématiques, auraient été normalisés par leur incorporation au sein du programme de recherche darwinien, de la même manière que d'autres éléments du débat actuel portant sur l' «évo-dévo» seraient en voie de l'être.

La part véritablement originale de la thèse promue par Barbara Continenza tient à la dimension des rapports entre science et métaphysique qu'elle y ajoute. Non seulement une portion significative des vues scientifiques de Waddington ont fini par s'imposer, insiste-t-elle, mais l'inspiration ultime de ces mêmes éléments scientifiques est à puiser du côté de sa métaphysique, une métaphysique présentant des similitudes avec celles des fondateurs de la Synthèse moderne:

Que de points de contact entre cette métaphysique et celles de Huxley, Dobzhansky, Rensch! Le progrès, la direction de l'évolution, l'éthique, le rôle de l'homme [...]. L'argument est le suivant: la métaphysique de Waddington semblerait avoir constitué le fonds effectif d'un réel projet de recherche empirique et scientifique qui est concrétisé et s'est intégré au noyau de la théorie de l'évolution $[\ldots]$.

Toutefois, il n'est pas clair que Barbara Continenza entende défendre l'idée que les métaphysiques de Huxley, Dobzhansky, Rensch seront éventuellement intégrées au darwinisme. Chose certaine, elle se fait la promotrice d'un programme de recherche darwinien aux capacités d'intégration, citant pour ce faire les mots de Jean Gayon: «[...] les traditions demeurent et se transmettent dans le mouvement de leur intégration dans de nouveaux patchworks, indéfiniment".

La thèse de Barbara Continenza soulève une question capitale pour la compréhension de la nature du darwinisme: bien que nous admettions parfaitement avec elle que le mouvement darwinien possède une capacité bien réelle d'intégration des éléments extérieurs à lui-même dans la durée, cette capacité d'absorption est-elle illimitée? Dans l'éventualité d'une réponse positive, il faudrait concevoir la trame darwinienne comme essentiellement 
linéaire: après la formulation initiale de la théorie sélective sur la base de variations organismiques a suivi la phase y intégrant les réalités génétiques, elle-même suivie par celle présentement en cours, occupée à intégrer la biologie développementale. Il faut le reconnaître, ce modèle linéaire est séduisant. Or la cohérence cognitive interne au darwinisme peut-elle se trouver menacée devant certains enjeux conceptuels, ontologiques et métaphysiques? Dans un ouvrage remarquable, Darwinism Evolving (1995), David Depew et Bruce Weber poussent l'idée de l'élasticité conceptuelle du darwinisme encore plus avant. Définissant le darwinisme comme une tradition de recherche ayant pour noyau le concept de la sélection naturelle, ces auteurs défendent que celle-ci a continuellement renouvelé, depuis Darwin, l'ontologie au cœur de la dynamique évolutive: d'abord par la dynamique newtonienne, ensuite par la dynamique boltzmannienne, et plus récemment par celle faisant appel aux systèmes complexes non linéaires. C'est ainsi que de très nombreux développements pertinents à l'évolutionnisme sont incorporés au sein du darwinisme, ceux tirés de la génétique des populations et moléculaire, de la biologie développementale et organismique, de la thermodynamique et des propriétés auto-organisatrices de la matière.

La cohabitation de tous ces enjeux épistémologiques - de méthodes, de concepts, de théories, d'ontologies - au sein d'un darwinisme que l'on veut de plus en plus généreux et intégré à tout moment me semble introduire de réelles tensions pour la cohérence et la cohésion du darwinisme. Nous savons ce qu'il est advenu de "la grenouille qui veut se faire aussi grosse que le bœuf » dans la fable de Jean de La Fontaine. Il semble que la thèse de Depew et Weber conçoive le développement du darwinisme comme essentiellement une affaire de substitution dans l'ontologie du processus, soit les changements successifs dans l'explication de la dynamique évolutive ${ }^{7}$. Or il me semble que ce parcours implique aussi le «(sur)chargement» du darwinisme, si l'on peut dire, l'entraînant sur le chemin de l'ontologie de la matière de plus en plus profonde: si Darwin applique la dynamique newtonienne aux variations organismiques, la phase boltzmannienne subséquente incorpore à cette dynamique évolutive les gènes et les molécules complexes, alors que l'étape actuelle interpelle la dynamique des systèmes complexes non linéaires dont le fondement est également pertinent pour l'étude de la matière dite «inerte» (les molécules simples). Le maintien ici d'une distinction entre l'ontologie du processus et l'ontologie de la matière me semble illusoire puisque ne pouvant simplement reposer sur un emprunt métaphorique ou une valeur heuristique. Si les réseaux génétiques présentent des complexités imprévues pouvant peut-être s'expliquer par la théorie du chaos ${ }^{8}$, c'est aussi parce qu'ils sont reliés d'une façon ou d'une autre aux processus sous-jacents de la matière inerte (voir aussi mes réponses aux commentaires de Michel

7. Depew et Weber, 1995, p. 2-4, 25-26, 29-30.

8. Ibid., 3. 
Morange). Certainement, l'émergence de réalités même nouvelles et imprévisibles aux niveaux supérieurs ne peut se faire au détriment complet des réalités inférieures, comme par une sorte de transcendance toute platonicienne. Après tout, les études évolutionnistes ne sont pas séparées en deux par un schisme comme c'est le cas pour la physique quantique (l'infiniment petit) et la physique relativiste (l'infiniment grand). Il est question ici d'une échelle relativement graduelle de la matière: organismes, gènes, molécules complexes et molécules simples. Il était à prévoir que la superposition de tous ces niveaux hiérarchiques ou ontologiques de la matière dans le darwinisme mènerait à de réels tensions et problèmes explicatifs. Le vieux problème concernant la nature de la trilogie disciplinaire biologie-chimiephysique me semble incontournable.

Pour ma part, je propose une interprétation alternative qui consiste à éviter la surenchère en faveur d'un darwinisme toujours plus inclusif. J'avance la thèse radicale suivante: il n'existe pas à proprement parler de paradigme ou de programme de recherche darwinien; seule existe la théorie de la sélection naturelle originellement proposée par Charles Darwin et depuis intégrée aux divers programmes de recherche transformistes souvent concurrents - parfois complémentaires - évoluant en parallèle depuis le $\mathrm{XIX}^{\mathrm{e}}$ siècle, notamment ceux fondés sur l'évolution cosmique (universelle), la biologie développementale, la thermodynamique, l'auto-organisation, etc. À l'exception des savants cherchant à développer la théorie de la sélection naturelle elle-même, l'essentiel de l'effort évolutionniste est tourné vers ces autres programmes de recherche au sein desquels l'intégration de la théorie sélective ne constitue qu'un élément parmi d'autres. Ainsi, le concept de la sélection naturelle ne constitue pas le noyau central autour duquel ces programmes de recherche sont élaborés. C'est donc sans surprise que l'on constate que la notion de sélection naturelle subit des modulations conceptuelles selon les programmes de recherche concurrents au sein desquels elle se trouve insérée: l'effort de cohérence et d'harmonisation est à cette condition. D'ailleurs, il serait faux de croire que seuls Darwin et ses successeurs directs ont le monopole de la bonne utilisation du concept de la sélection naturelle. $\mathrm{Vu}$ de cette perspective, le "darwinisme", qu'il ne faut pas confrondre avec la théorie de la sélection naturelle, est davantage une vague appellation à l'appui d'une théorie sélective en évolution biologique plus que le siège unique d'un lieu où s'effectue une réflexion sur celle-ci. Peut-être devrait-on aller jusqu'au bout du raisonnement et suggérer que l'on banisse tout simplement l'étiquette de «darwinisme».

Cette thèse radicale me semble avoir le mérite d'offrir une explication élégante à une situation tout à fait inattendue: le conflit entre plusieurs fondateurs de la Synthèse moderne et cette même théorie qu'ils tentent d'établir! En effet, j'avance ailleurs l'idée que la Synthèse moderne s'est avérée être une sorte de piège théorique puisqu'elle ne convient pas parfaitement aux divers évolutionnismes en voie d'élaboration par ces fon- 
dateurs ${ }^{9}$. Ainsi: Huxley s'est vu obligé de rester indifférent aux inconsistances se faisant jour entre sa vision cosmique de l'évolution et sa propre lecture des mécanismes néo-darwiniens; Simpson n'a pu parvenir à harmoniser l'action de ces mêmes mécanismes avec les fortes directionalités qu'il a lui-même observées dans les annales de la vie; Dobzhansky et Rensch ont dû distordre le concept de la sélection naturelle afin de lui faire jouer le rôle moteur directionnel que leurs visions cosmiques respectives exigeaient. En réalité, les fondateurs de la Synthèse moderne ont instrumentalisé la théorie de la sélection naturelle au profit de programmes de recherche concurrents faisant la promotion de visions évolutionnistes variablement fondées sur: 1) des épistémologies/métaphysiques du devenir ou des origines; 2) des approches historiciste ou étiologique; et 3) des conceptions cosmique ou biologique. Je crois qu'il n'est pas exagéré dans ces circonstances d'user de l'expression kuhnienne d' «incommensurable » pour qualifier les visions des fondateurs de la Synthèse moderne. Pour le dire ainsi, ces fondateurs sont moins darwiniens que l'on imagine, et la trame menant de Darwin à aujourd'hui est sinueuse. À mon sens, la notion d'une voie royale unique pour les études darwiniennes - le programme de recherche dit darwinien - me semble contreproductive pour la compréhension des études évolutionnistes tant générales que darwiniennes.

Ultimement, certes, il ne peut y avoir qu'une seule vraie théorie de l'évolution, qu'un seul programme de recherche véridique portant sur l'évolution. Seulement, le chemin complexe et difficile pour y parvenir requiert à ce stade-ci, me semble-t-il, l'existence de voies exploratoires multiples par l'intermédiaire de programmes de recherche parallèles. Peut-être l'un d'eux parviendra-t-il à triompher seul des autres, bien qu'il soit plus probable qu'une synthèse basée sur la sélection de certains éléments apparemment contradictoires émergera victorieuse. Dans cette éventualité, il est essentiel d'encourager l'établissement d'une réflexion multidisciplinaire et l'érection de ponts interdisciplinaires.

\section{Réponse au commentaire de Michel Morange}

Mon étude des fondateurs de la Synthèse moderne ne constitue pas formellement une entrée de ma part dans le débat théorique contemporain. Il serait faux de croire que je m'exprime par personnes interposées. Ma stratégie épistémologique - et c'en est une - est ailleurs: le pluralisme inhérent, trop longtemps méconnu chez certains fondateurs de la Synthèse moderne, est suffisamment révélateur et riche en enseignements pour éclairer une certaine étroitesse de vues se faisant jour chez les penseurs des générations postérieures. Le confinement des débats récents en biologie de l'évolution repose sur une base relativement consensuelle dont le fondement me semble

9. Delisle, 2011, p. 57. 
être poreux, voire illusoire. Je m'inquiète d'une biologie de l'évolution fermée sur elle-même, forte de la certitude que les débats évolutionnistes n'ont de pertinence qu'en son sein propre. Cette attitude est pour le moins surprenante à l'ère où le principe unificateur le plus puissant est peut-être l'évolutionnisme universel tel qu'incarné dans la théorie du Big Bang.

Ce que j'ai trouvé chez les fondateurs de la Synthèse moderne, c'est une conscience aiguë d'une science véritablement universelle adaptée à l'ampleur que requiert la question de l'unification en marche des divers évolutionnismes (cosmologique, biologique, culturel). Cette conscience est accompagnée d'un pluralisme épistémologique non seulement sain mais tout simplement nécessaire face à la tâche colossale que constitue le chantier de l'évolutionnisme général. Le chemin parcouru à ce jour, me semblet-il, n'est rien en regard de celui qui reste à parcourir. Ainsi, si je n'ai pas à souscrire à la lettre des travaux des fondateurs de la Synthèse moderne, c'est sans réserve aucune que j'embrasse l'esprit qui les animait. Cela dit, je ne reculerai pas devant mes obligations et répondrai à Michel Morange sur le terrain scientifique et épistémologique, d'autant que nous sommes tous deux motivés par l'idée d'un réquisitoire contre une certaine pensée unique en biologie de l'évolution. Toutefois, ma démarche s'inscrit moins dans la recherche de réponses que dans la création des conditions propices pour y accéder.

\section{La Synthèse moderne: origine révolutionnaire et suite consensuelle?}

Plusieurs biologistes, historiens et philosophes s'accorderaient avec Michel Morange sur l'idée que le champ de la biologie de l'évolution se trouve aujourd'hui à un stade relativement consensuel de son développement. "Parmi les évolutionnistes, les opinions divergentes sont devenues rares", insiste-t-il. Cette thèse soulève une question cruciale: qu'est-il advenu des divergences épistémologiques, ontologiques, et métaphysiques incommensurables proposées par les fondateurs de la Synthèse moderne comme Julian Huxley, Theodosius Dobzhansky, Bernhard Rensch, George Simpson et Ernst Mayr, auteurs s'exprimant encore là-dessus dans les années 1970 ou 1980 (ils sont tous morts entre 1975 et 2005)? Quoiqu'il reconnaisse que certains de ces points de vue aient été "poussés sous le tapis", Michel Morange semble privilégier la thèse que la Synthèse moderne a éventuellement produit un "effet unificateur ", forgeant ainsi un socle théorique relativement consensuel. Pour ma part, je m'interroge sur la raison de la naissance soudaine de cet apparent consensus à la suite d'une période (19401960) que Michel Morange qualifie très justement de «révolutionnaire » - reprenant la terminologie kuhnienne - afin de marquer toute l'ampleur de la transition d'une période à l'autre.

En fait, cette transition si profonde me semble requérir une autre explication: la spécialisation, voire la surspécialisation, de la formation scientifique. Ici, on pourrait m'accuser de recourir à un subterfuge sociolo- 
gique afin de contourner une question cognitive ou théorique. Or il n'en est rien. Les avancées de la science créent un impact tout à fait tangible sur la formation des chercheurs, à un point tel que la réussite se fait souvent de nos jours aux dépens de la formation généraliste. C'est ainsi que l'ensemble des questions concernant la biologie de l'évolution, croit-on, se résoudra par des biologistes de l'évolution avec la modeste participation des historiens et des philosophes de la «biologie», eux-mêmes pris dans le sillage de la spécialisation. Il faut le dire clairement, l'ampleur de la formation des fondateurs de la Synthèse moderne lors du premier tiers du $\mathrm{xx}^{e}$ siècle est sans commune mesure avec celle des générations récentes. Croire que cette différence institutionnelle n'y est pour rien dans notre affaire me semble tout à fait illusoire. J'en veux pour preuve certains travaux récents aux implications évidentes pour la réflexion évolutionniste, et trop souvent laissés pour compte par les penseurs de la biologie de l'évolution: Wicken, Evolution, Thermodynamics, and Information (1987); Brooks et Wiley, Evolution as Entropy (1988); Goodwin, How the Leopard Changed Its Spots (1994); Kauffman, At Home in the Universe (1995). Ces travaux portent principalement sur l'implication des principes de la thermodynamique et de l'autoorganisation en évolution. Si je n'ai pas à souscrire à l'ensemble de ces thèses — ce n'est d'ailleurs pas le cas - il n'empêche que l'imperméabilité de la biologie de l'évolution à l'endroit de ces thèses est révélatrice de frontières qui tiennent davantage à la spécialisation qu'à l'impertinence de celles-ci pour la biologie. Et pourtant, c'est au cœur de cette autre littérature que se font jour les points de vue véritablement divergents sur l'évolutionnisme. La "pensée unique» en biologie de l'évolution, pour reprendre les mots de Michel Morange, me semble davantage être le produit de la surspécialisation que de l'effet unificateur de la Synthèse moderne.

\section{L'évolution cosmique (universelle)}

Les travaux énumérés ci-haut ancrent de fait l'évolutionnisme dans l'évolution cosmique. Or Michel Morange nous dit qu'il a «beaucoup de mal cependant à voir comment une théorie de l'évolution cosmique viendrait expliquer l'évolution des formes vivantes». D'abord, il conviendrait peutêtre d'écarter un obstacle verbal en remplaçant l'expression d' "évolution cosmique » par celle d' "évolution universelle» afin de dissiper tout malentendu concernant de possibles évocations mystiques. Parler d'évolution universelle, c'est tout simplement s'intéresser à l'évolution de la matière. Dans la mesure où, d'une part, la matière terrestre est très largement représentative des éléments échantillonnés ailleurs dans l'univers et, d'autre part, que le grand principe organisateur et unificateur en voie de s'imposer en cosmologie est celui de l'évolution elle-même (la théorie du Big Bang), il semble difficile d'échapper à la conclusion qu'il existe un lien quelconque entre matière inerte et matière organique. Certainement, l'impulsion donnée à l'origine du processus d'évolution biologique est partiellement tributaire du 
processus d'organogenèse au sein de la matière dite «inerte». On en veut pour preuve les vertus auto-organisatrices d'éléments inertes (les molécules simples) s'organisant spontanément en entités plus complexes. "Order for free ", dira Stuart Kauffman ${ }^{10}$. Nul doute qu'il s'agit là d'une forme des plus primaires d'évolution; peut-être est-ce même abuser du terme. N'empêche qu'il n'est plus possible aujourd'hui d'opposer de manière absolue la sphère de la matière inorganique et celle de la matière organique.

Certes, cela soulève la délicate question des rapports entre les sciences physico-chimiques et les sciences biologiques sous la forme d'une double dichotomie: déterminisme/contingence; réductionnisme/holisme. L'époque d'une science physique toute conquérante imposant tant son déterminisme que son réductionnisme est révolue. D'abord parce que le holisme et la contingence ont depuis fait leur entrée au cœur des sciences physiques (la théorie du chaos), mais ensuite et surtout parce que la biologie de l'évolution a conquis de haute lutte la reconnaissance d'une réalité propre au niveau de la matière organique, qu'il s'agisse de la part de contingence exprimée dans les capricieuses ramifications de l'arbre de la vie ou encore de l'impossibilité de vider les questions de biologie organismique par le recours exclusif aux entités constituantes inférieures (réductionnisme épistémologique). Voilà pourquoi la notion d' 'émergence» s'est imposée en biologie de l'évolution comme principe fondateur à ce niveau de la hiérarchie de la matière.

Cela dit, l'avenir de l'évolutionnisme va se jouer à mon avis dans la zone grise se situant à l'interface de l'inerte et de l'organique. D'ailleurs, parler d'interface est trompeur puisque cela donne une fausse impression de contact entre deux réalités. Or nous n'en sommes plus là depuis un moment. Il faudrait plutôt penser en termes d'épaisseur, soit de multiples niveaux d'intégration aux propriétés différentes et mal comprises, mais emboîtés les uns dans les autres au sein d'une sorte de continuum allant de la matière inerte à la matière organique. Dans un sens, la «boîte noire» de Charles Darwin n'est plus. C'est-à-dire que la génétique des populations des années 1920 et 1930 a répondu aux incertitudes et aux incompréhensions que Darwin soulevait sur la nature du processus de transmission du matériel génétique par-delà les générations. Et pourtant, la quête se poursuit dans une sorte de régression à l'infini de la source toujours plus ultime de l'information encodée et de ses propriétés d'expression et de transmission. Les tentatives assez récentes décrites par Michel Morange pour mieux saisir l'interaction entre la biologie fonctionnelle et la biologie évolutionnaire afin de comprendre «la longue chaîne causale qui relie le génotype et le phénotype $^{11}{ }^{\prime}$ s'inscrivent précisément dans cette démarche. Faut-il s'arrêter en si bon chemin? Je ne le crois pas. Une explication scientifique totale et une

10. Kauffman, 1995 , p. 71.

11. Voir aussi Morange, 2011. 
synthèse complète se doivent de s'enfoncer encore plus profondément dans la matière. C'est d'ailleurs l'objectif de la démarche si mal comprise de Bernhard Rensch, et que j'ai tenté de restituer. Aussi étrange que cela puisse paraître à nos esprits nourris de dichotomies, il semble bien que la contingence dans la matière organique ait trouvé le moyen d'émerger d'un déterminisme au cœur de la matière inerte. Parce que l'avenir est appelé à poursuivre la dissolution de l'apparente étanchéité de la cloison entre l'organique et l'inorganique, je vois mal comment l'évolution biologique et l'évolution universelle (cosmique) pourraient demeurer totalement distinctes, tant dans l'ordre disciplinaire que dans l'ordre épistémologique. Reste ensuite à établir la véritable nature des similitudes et des différences.

Après réflexion, peut-être que mes vues ne diffèrent de celles de Michel Morange que par le regard que nous portons sur l'échéancier de la recherche: il me semble évident que des gains tangibles et durables viendront d'abord du côté de l'analyse des rapports entre la biologie fonctionnelle et la biologie évolutionnaire. Il faudra attendre plus longtemps les résultats concernant la compréhension de la matière plus profonde. Mais il ne faudrait pas confondre les limites de l'enquête immédiate avec les impossibilités de principe. C'est ainsi que certains penseurs plus hardis (ou impatients) ont déjà entamé une réflexion sur la sélection naturelle dans le cadre de la théorie du Big Bang: Smolin, The Life of the Cosmos (1997); Davis, The 5th Miracle (2000); Impey, The Living Cosmos (2007). N'en déplaise aux biologistes de l'évolution, l'objet d'étude qu'ils croient leur être exclusif s'en trouve déjà investi par des chercheurs venus d'ailleurs, notamment de la cosmologie. Afin d'éviter les abus de toutes sortes, il serait peut-être judicieux de s'en mêler. Voilà peut-être pourquoi plusieurs fondateurs de la Synthèse moderne avaient ressenti la nécessité d'occuper le terrain. Les absents ont toujours tort.

\section{Causes efficientes et causes dites «finales》 (actualisme et historicisme)}

Nulle part ailleurs mes propos auront été plus mal compris que sur la question des causes dites «finales". Michel Morange écrit : "[Delisle] fait remarquer que rien ne prouve que la science de demain privilégiera autant que celle d'aujourd'hui les mécanismes, et refusera toute place aux causes finales. " Non seulement mes travaux ne font aucunement la promotion des causes finales ou de la téléologie cosmique, mais il en est de même pour ceux de Huxley, Dobzhansky, Rensch, Simpson, et Mayr qui parlent de directionalité évolutive observée descriptivement, ce qui n'est pas du tout la même chose. Je suis d'accord avec Michel Morange lorsqu'il dit que «l'abandon des causes finales me semble être un phénomène irréversible ", et j'irais même plus loin en affirmant que la quête des causes finales ou téléologiques s'inscrit à l'extérieur d'une démarche proprement scientifique. La seule exception, fort circonscrite d'ailleurs, est celle des processus dits téléonomiques (dans la terminologie de Mayr), et qui sont communs dans la sphère du vivant pour des processus conçus pour l'atteinte d'un but comme la 
migration saisonnière, la recherche de nourriture, la reproduction, le développement ontogénétique, etc. Puisque ces restrictions concernant la question des «causes finales» sont largement admises par la communauté scientifique, il est inutile d'insister davantage.

C'est ainsi que toute la question de l'opposition entre les causes efficientes et les causes incorrectement nommées «finales» se situe à un autre niveau d'explication et met en scène de véritables enjeux épistémologiques trop souvent négligés ou mal compris. Depuis le début de la révolution transformiste au tournant des XVIII ${ }^{\mathrm{e}}$ et $\mathrm{XIX}^{\mathrm{e}}$ siècles, la quête transformiste a été investie de deux manières différentes. Épistémologiquement parlant, deux approches - parfois en contradiction, parfois en complémentarité ont été utilisées pour générer de la connaissance en évolution. Historiquement la plus ancienne, l'approche historiciste ou l'historicisme (entendu ici dans un autre sens que celui de Karl Popper) a cherché à étoffer l'évolution dans toute sa matérialité, dans toute son ampleur et sa réalité empirique: la série animale dans le présent, l'arbre de la vie dans les temps géologiques, le développement ontogénétique, les étapes de la cosmogenèse, etc. De manière générale, l'historicisme - plus descriptif qu'explicatif - révèle toute la dimension temporelle ou verticale de l'évolution. L'autre approche se détourne de la temporalité et du descriptif au profit des causes efficientes. Celle-ci est fondée dans l'actualisme, c'est-à-dire qu'il s'agit essentiellement de comprendre les mécanismes et les processus de l'évolution par l'étude de phénomènes actuels (ou horizontaux).

Parfois traitées de manière exclusive, ces deux approches sont aussi considérées simultanément par plusieurs penseurs comme cela est le cas chez les fondateurs de la Synthèse moderne. Et c'est de la confrontation des approches actualiste et historiciste que naissent souvent les difficultés les plus grandes pour les études évolutionnistes. En effet, il arrive parfois que leur rencontre soit brutale du fait qu'elles semblent mener à des résultats contradictoires. Par exemple, que faire si les causes efficientes darwiniennes (la théorie de la sélection naturelle) semblent indiquer l'importance du hasard et de la contingence dans le processus évolutif alors même qu'une interprétation donnée à l'arbre de la vie favorise une directionalité suffisamment forte pour mettre en cause la prédominance de cette contingence? La résolution empirique de cette question n'est pas mon propos ici; seules les implications épistémologiques m'intéressent. Faut-il prioriser les causes efficientes au détriment d'une connaissance plus descriptive issue de l'historicisme, ou l'inverse? Sur ce point précis, mon avis diverge de celui de Michel Morange qui écrit:

L'observation des progrès "évidents» des organismes n'a guère de poids devant un mécanisme causal à la vertu explicative largement démontrée [...] Il y a, à l'heure actuelle, un écart infranchissable entre l'observation vague de tendances progressives dans l'évolution, et la possibilité de proposer des processus causaux pour les expliquer. 
Et pourtant, le néo-darwinisme des causes efficientes a reçu une leçon en règle de la part des paléontologistes Niles Eldredge et Stephen Jay Gould lors du débat portant sur la théorie des équilibres ponctués dans les années 1970 et 1980. Il a été démontré qu'une portion significative des archives fossiles avait enregistré des stagnations évolutives prolongées suivies de sauts brusques, et ce, contrairement à la prédiction de la théorie qui voulait que les transformations devaient se produire selon un processus essentiellement continu et graduel. L'espace me manque ici pour entrer dans les subtilités du débat. Mon propos est le suivant: la connaissance descriptive issue de l'approche historiciste (la paléontologie dans ce cas-ci) a clairement démontré lors de cet épisode son utilité foncière pour corriger des choix conceptuels et méthodologiques tirés de l'actualisme, notamment l'ontologie excessivement atomistique au cœur du néo-darwinisme et son réductionnisme négateur des réalités organismiques, choix issus d'une survalorisation de l'approche génétique (moléculaire ou populationnelle).

Parce que l'évolution est, par définition, un objet d'étude qui se déploie dans la temporalité — c'est même l'objet temporel par excellence —, il existera toujours un risque réel que l'étude des causes efficientes dans le moment présent puisse rester aveugle à des phénomènes dont les manifestations sont évanescentes à cette échelle. Affirmer le contraire serait pousser la préséance de l'épistémologie actualiste sur l'épistémologie historiciste au-delà de sa véritable capacité de résolution des problèmes en évolution. Cela n'enlève rien à la puissance considérable de la connaissance des causes efficientes, surtout dans sa forme expérimentale puisque prédictible et reproductible. Mais la nature même de l'objet évolutionniste si furtif à l'actualité, et l'état incroyablement préliminaire de nos connaissances d'une synthèse évolutionniste véritablement universelle garantissent pour longtemps de beaux jours à l'historicisme. Selon moi, il est inutile de choisir entre ces deux épistémologies puisque leur complémentarité s'impose d'elle-même et, je dois ajouter, à égalité de force. Une fréquentation plus assidue des réalités empiriques propres à l'historicisme permettrait aux chercheurs de l'horizontalité (des causes efficientes) d'apprécier davantage les ressources étoffées propres à ces disciplines. Il nous faut simplement apprendre à vivre avec les tensions créatrices que génère la confrontation de ces deux épistémologies.

\section{Une biologie de l'évolution au stade pré-paradigmatique}

Au final, ce qui distingue fondamentalement la démarche de Michel Morange et la mienne semble tenir à la dimension variable que nous attribuons au terrain de jeu des évolutionnistes. C'est de manière tout à fait compréhensible qu'il écrit: "Parler d'un stade pré-paradigmatique pour la théorie actuelle de l'évolution me semble incorrect.» Il est vrai que, dans l'univers clos de la biologie de l'évolution, l'impression d'une théorie robuste et relativement consensuelle est bien réelle. Pour des raisons évoquées plus haut, je crois pour ma part qu'il est préférable de concevoir la révolution darwi- 
nienne comme une partie intégrante d'une révolution aux implications universelles: la révolution transformiste, elle-même porteuse du projet d'une synthèse des divers évolutionnismes (cosmologique, biologique et culturel). Puisque cette dernière révolution scientifique n'en est qu'à ses premiers balbutiements, il ne me semble pas déraisonnable de parler d'une biologie de l'évolution au stade pré-paradigmatique du simple fait que certains de ses présumés acquis empiriques, conceptuels, et théoriques restent menacés par des découvertes extérieures à ce champ disciplinaire. C'est ainsi que la question d'échelle séparant moi-même et Michel Morange ouvre sur d'intéressants choix épistémologiques. Alors que ce dernier s'en tient à un champ d'investigation plus restreint caractérisé par de patientes et minutieuses études - et il existe une foule de bonnes raisons de faire ce choix —, j'opte pour ma part pour un champ d'investigation élargi guidé par le principe qu'un problème vu par le prisme d'une échelle plus restreinte trouve parfois une solution différente à une échelle plus large. C'est ainsi que je prédis que la quête des principes régissant l'organisation au sein des entités évolutives ne parviendra pas à recouvrir toute la réalité par un recours exclusif à la biologie moléculaire, à la biologie cellulaire, etc. Les découvertes au sein de ces disciplines biologiques devront tôt ou tard composer avec celles émanant de la physique et de la chimie, c'est-à-dire que les interprétations respectives devront être modifiées dès lors qu'elles seront confrontées. C'est la difficile négociation du passage de l'inorganique (déterminisme) à l'organique (contingence) auquel nous avons déjà fait allusion. Il me semble opportun ici de mentionner la vision de la science promue par Otto Neurath et décrite dans les mots de Richard Creath:

Il faudrait que chacun écoute l'autre puisque la preuve que chacun avance pourrait être sans pertinence et être rejetée. La pertinence est après tout une voie à double sens. La théorie physique peut expliquer les observations biologiques, mais ces dernières peuvent tout aussi aisément détruire une théorie physique ou faire pencher la balance en faveur d'une théorie physique ou d'une autre. Quoi qu'il en soit, l'unité ne peut pas être imposée a priori. Elle émerge uniquement dans la mesure où elle renforcerait les théories provenant des diverses disciplines. D'après moi, c'est le principe d'unité suscitée par la coopération mis de l'avant par Neurath ${ }^{12}$.

Deux approches du progrès scientifique sont donc concevables: la simple addition des connaissances tirées d'une foule de champs disciplinaires, ou l'interaction des savoirs dans l'élaboration même de ces connaissances. Personnellement, je crois que le tout (la synthèse des connaissances) est plus que la somme de ses parties (l'addition des savoirs disciplinaires indépendants). C'est ce que l'on pourrait appeler un holisme épistémologique.

12. Creath, 1996, p. 168-169. 


\section{Bibliographie}

Becquemont, Daniel. Charles Darwin, 1837-1839: Aux sources d'une découverte, Paris, Kimé, 2009.

Brooks, Daniel et Edward Wiley. Evolution as Entropy: Toward a Unified Theory of Biology, 2e édition, Chicago, University of Chicago Press, 1988.

Creath, Richard. "The Unity of Science: Carnap, Neurath, and Beyond». In P. Galison et D. J. Stump (dir.), The Disunity of Science: Boundaries, Contexts, and Power, Stanford, Stanford University Press, 1996, p. 158-169.

Darwin, Charles. On the Origin of Species, London, Murray, 1859.

Davis, Paul. The 5th Miracle: The Search for the Origin and Meaning of Life, New York, Touchstone, 2000.

Delisle, Richard. «What Was Really Synthesized During the Evolutionary Synthesis? A Historiographic Proposal». In R. Delisle (dir.), Defining Darwinism: One Hundred and Fifty Years of Debate, special issue of Studies in History and Philosophy of Biological and Biomedical Sciences 42, 2011, 50-59.

- Les philosophies du néo-darwinisme: Conceptions divergentes sur l'homme et le sens de l'évolution, Paris, Presses Universitaires de France, 2009.

Depew, David et Bruce Weber. Darwinism Evolving: Systems Dynamics and the Genealogy of Natural Selection, Cambridge, MIT Press, 1995.

Dobzhansky, Theodosius. Genetics and the Origin of Species, New York, Columbia University Press, 1937.

Eldredge, Niles. Unfinished Synthesis: Biological Hierarchies and Modern Evolutionary Thought, New York, Oxford University Press, 1985.

Gayon, Jean. «Neo-Darwinism». In G. Wolters G. et J. G. Lennox (dir.), Concepts, Theory and Rationality in the Biological Sciences, Konstanz, Konstanz University Press and Pittsburgh University Press, 1995, p. 1-25.

Goodwin, Brian. How the Leopard Changed Its Spots: The Evolution of Complexity, Princeton, Princeton University Press, 1994.

Gould, Stephen. «Is a New and General Theory of Evolution Emerging ? ", Paleobiology 6, 1980, 119-130.

Hull, David. Darwin and His Critics, Cambridge, Harvard University Press, 1973.

Huxley, Julian. Evolution: The Modern Synthesis, New York, Harper \& Brothers, 1942.

Impey, Chris. The Living Cosmos: Our Search for Life in the Universe, New York, Random House, 2007.

Kauffman, Stuart. At Home in the Universe: The Search for Laws of Self-Organization and Complexity, Oxford, Oxford University Press, 1995.

Kuhn, Thomas. The Structure of Scientific Revolutions, $2^{\mathrm{e}}$ édition, Chicago, University of Chicago Press, 1970.

Lakatos, Imre. "Falsification and the Methodology of Scientific Research Programmes ». In I. Lakatos et A. Musgrave (dir.), Criticism and the Growth of Knowledge, Cambridge, Cambridge University Press, 1970, p. 91-196.

Mayr, Ernst. Systematics and the Origin of Species, New York, Columbia University Press, 1942.

—. "Teleological and Teleonomic: A New Analysis». In R. S. Cohen et M. W. Wartofsky (dir.), Methodological and Historical Essays in the Natural and Social Sciences, Dordrecht, D. Reidel, 1974, p. 91-117. 
Morange, Michel. «What Will Result From the Interaction Between Functional and Evolutionary Biology?». In R. Delisle (dir.), Defining Darwinism: One Hundred and Fifty Years of Debate, special issue of Studies in History and Philosophy of Biological and Biomedical Sciences 42, 2011, 69-74.

Rensch, Bernhard. Neuere Probleme der Abstammungslehre: Die transspezifische Evolution, Stuttgart, Enke, 1947.

Simpson, George. Tempo and Mode in Evolution, New York, Columbia University Press, 1944.

Smocovitis, Vassiliki Betty. Unifying Biology, Princeton, Princeton University Press, 1996.

Smolin, Lee. The Life of the Cosmos, Oxford, Oxford University Press, 1997.

Wicken, Jeffrey. Evolution, Information and Thermodynamics: Extending the Darwinian Program, Oxford, Oxford University Press, 1987. 